Interesting image

\title{
Sigmoid colon metastasis from metaplastic breast carcinoma mimicking primary sigmoid colon cancer
}

\section{Metástasis en el colon sigmoide de un carcinoma de mama imitando cáncer de colon sigmoide primario}

\author{
H.W. Kim ${ }^{\mathrm{a}, *}$, D.H. Moon ${ }^{\mathrm{b}}$ \\ a Department of Nuclear Medicine, Keimyung University, School of Medicine, Daegu, South Korea \\ ${ }^{\mathrm{b}}$ Department of Nuclear Medicine, Asan Medical Center, University of Ulsan College of Medicine, Seoul, South Korea
}

\section{A R T I C L E I N F O}

\section{Article history:}

Received 23 July 2014

Accepted 3 September 2014

Available online 18 October 2014

A 46-year-old woman had undergone breast conserving operation for right breast cancer with no evidence of distant metastasis. The histopathological examination revealed a matrix-producing metaplastic breast carcinoma, and all eight resected axillary lymph nodes were negative for carcinoma. Immunohistochemistry

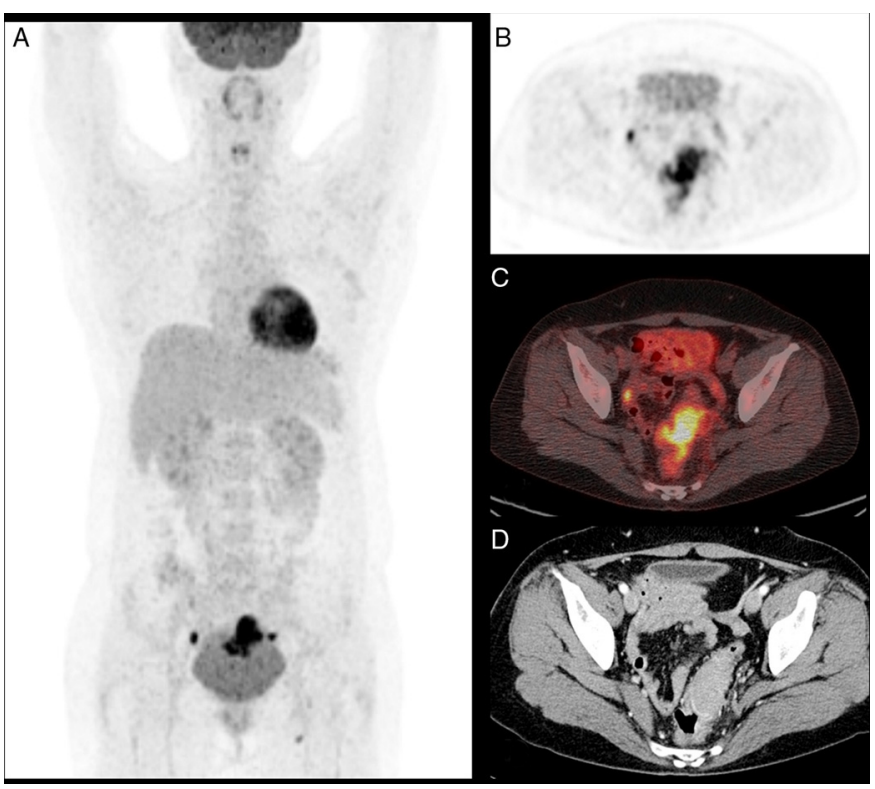

Fig. 1. Maximum intensity projection (A), PET (B), and fusion (C) images of PET/CT show a hypermetabolic mass in the sigmoid colon (SUVmax 9.7). Contrast-enhanced CT (D) shows eccentric wall thickening of the distal sigmoid colon with an enhanced soft tissue density mass.

* Corresponding author at: Department of Nuclear Medicine, Keimyung University, School of Medicine, 194 Dongsan-Dong, Jung-Gu, Daegu 700-712, South Korea.

E-mail address: yapp99@hanmail.net (H.W. Kim).
(IHC) staining showed that the right breast cancer was negative for estrogen receptor (ER), progesterone receptor (PR), and human epidermal growth factor receptor-2 (HER-2). The patient received adjuvant chemotherapy and radiotherapy. After two years, the patient developed intermittent hematochezia, and ${ }^{18} \mathrm{~F}$ fluorodeoxyglucose (FDG) whole-body PET/CT scan was performed.

The patient fasted for at least $6 \mathrm{~h}$ prior to the PET/CT examination and presented a blood glucose level of $97 \mathrm{mg} / \mathrm{dL}$. Image acquisition was performed after $1 \mathrm{~h}$ after the intravenous injection of $370 \mathrm{MBq}$ of ${ }^{18}$ F-FDG.

The ${ }^{18}$ F-FDG PET/CT showed a hypermetabolic mass in the sigmoid colon (Fig. 1). The contrast-enhanced CT showed eccentric wall thickening of the distal sigmoid colon with an enhanced soft tissue density mass. The colonoscopy showed a protruding mass above the anal verge. The histopathological examination of the colonoscopic biopsy specimen revealed poorly differentiated carcinoma similar to that of the previous metaplastic breast carcinoma (Fig. 2). IHC staining demonstrated that the sigmoid colon cancer was negative for CK20, CK5/6, ER, PR, and HER-2, but positive for

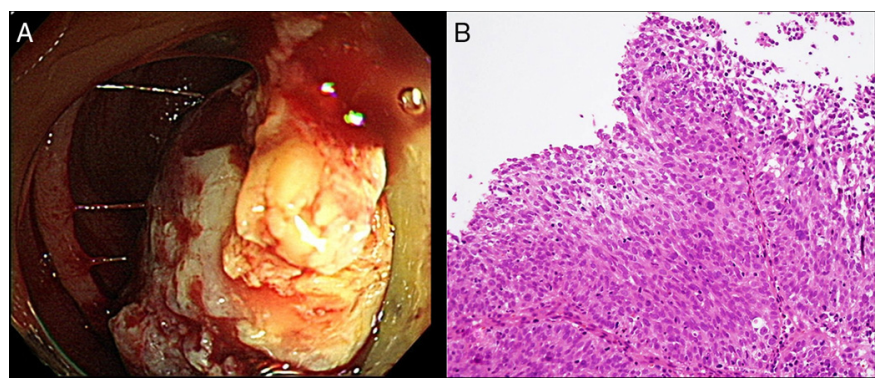

Fig. 2. Colonoscopy (A) shows a protruding mass at $11 \mathrm{~cm}$ above the anal verge taking up approximately half of the intestinal lumen. The histopathological examination (B) of the colonoscopic biopsy specimen reveals poorly differentiated carcinoma, which was similar to that of the previous metaplastic breast carcinoma (hematoxylin and eosin stain; original magnification 200). The sigmoid colonic lesion was diagnosed to be a metastasis from the metaplastic breast carcinoma. 
CK7. Therefore, the sigmoid colonic lesion was diagnosed to be a metastasis from the metaplastic breast carcinoma.

Metaplastic breast carcinoma is a rare, heterogeneous disease comprising $1 \%$ of all the invasive breast cancers. ${ }^{1}$ Most metaplastic breast carcinomas are poorly differentiated, high grade, and of triple-negative receptor status with a high hematogenous metastatic potential to extranodal sites such as lung and bone. ${ }^{1}$ There have been a few reports of colonic metastasis of invasive ductal or lobular breast carcinomas ${ }^{2,3}$, but colorectal metastasis from the metaplastic breast carcinoma has not been reported to the best of our knowledge. The metastatic breast cancer to the sigmoid colon can be easily misdiagnosed as primary sigmoid colon cancer. In the patients with breast cancer, the diagnosis of the colorectal metastasis is difficult not only because it is infrequent but also because of its nonspecific clinical presentation and variable radiographic features. ${ }^{3}$ Physicians should increase their clinical awareness in that any patient with a colorectal lesion and a history of malignancy should be considered to have a metastasis until proven otherwise.

\section{References}

1. Tse GM, Tan PH, Putti TC, Lui PC, Chaiwun B, Law BK. Metaplastic carcinoma of the breast: a clinicopathological review. J Clin Pathol. 2006;59:1079-83.

2. Zhou XC, Zhou H, Ye YH, Zhang XF, Jiang Y. Invasive ductal breast cancer metastatic to the sigmoid colon. World J Surg Oncol. 2012;10:256.

3. Bamias A, Baltayiannis G, Kamina S, Fatouros M, Lymperopoulos E, Agnanti N, et al. Rectal metastases from lobular carcinoma of the breast: report of a case and literature review. Ann Oncol. 2001;12:715-8. 